\title{
INITIAL COMMISSIONING OF THE ISAC RIB FACILITY
}

\author{
P.W. Schmor, TRIUMF, Vancouver, BC, Canada, V6T 2A3
}

\section{Abstract}

Construction began in 1995 on ISAC, a radioactive ion beam (RIB) and accelerator facility at TRIUMF that utilises the ISOL (on-line isotope separation) production method. ISAC includes: a new building, a beam line with adequate shielding to transport up to $100 \mathrm{uA}$ of $500 \mathrm{MeV}$ protons to two target/ion-source stations, remote handling facilities for the targets, a high-resolution mass-separator, linear accelerators and experimental facilities. The ISAC target/ion source station permits the production of nuclei far from stability over a large isotopic range with high luminosity. Ions from the target/ion-source can be transported at energies up to $60 \mathrm{keV}$ through a low-resolution pre-separator magnet followed by a high-acceptance, high-resolution mass-separator magnet to a variety of low energy experimental stations. Alternatively, ions with q/A greater than or equal to $1 / 30$ and an energy of $2 \mathrm{keV} / \mathrm{amu}$ can be bunched in the low energy beam transport line prior to a cw RFQ accelerator operating at $35 \mathrm{MHz}$. The $150 \mathrm{keV} / \mathrm{amu}$ beam from the RFQ is stripped and isotopes with a particular q/A greater than or equal to $1 / 6$ are selected for acceleration in a DTL. The DTL is a separated function accelerator with five accelerating tanks and three split-ring bunchers operating $\mathrm{cw}$ at $105 \mathrm{MHz}$. The final energy will be variable from 0.15 to $1.5 \mathrm{MeV} / \mathrm{amu}$. The accelerated beams will be used primarily for nuclear astrophysics studies. The beam commissioning of the proton beam line, target/ion source, mass separator and RFQ has started. The TRIUMF neutral atom trap (TRINAT) and a yield station began using the low energy ISAC beam in November 1998. The RFQ has accelerated stable ions up to $54 \mathrm{keV} / \mathrm{amu}$. The first tank of the drift tube linac and the subsequent buncher have been commissioned at full rf power. Beam commissioning of the DTL is scheduled for the end of 1999. The full-energy RIB will become available for the DRAGON recoil spectrometer at the end of 2000. A new five-year plan that includes an upgrade of ISAC to permit acceleration of radioactive ions up to 6.5 $\mathrm{MeV} / \mathrm{amu}$ for masses up to $150 \mathrm{amu}$ has been presented for funding.

\section{INTRODUCTION}

There have been more than ten years of experience with radioactive ion beams at TRIUMF using the TISOL facility [1]. TISOL is a first generation ISOL type facility that has not only provided useful beams for scientific research but has also provided valuable information on targets, ion sources and remote handling requirements for a second generation facility. Although the proton beam line for TISOL is capable of $10 \mu \mathrm{A}$, inadequate shielding above the target and primitive remote handling procedures for servicing irradiated components around the target have limited the proton beam intensity at TISOL to about $2 \mu \mathrm{A}$. TISOL will continue to be useful in the future as a facility to test new targets and ion sources for ISAC, albeit at lower currents. The design specifications for the ISAC-I RIB delivery system are listed in table 1 .

Table 1: Design Parameters for ISAC I

\begin{tabular}{|c|c|c|}
\hline \multicolumn{3}{|c|}{ ISOTOPE PRODUCTION SYSTEM } \\
\hline \multirow[t]{5}{*}{ Driver } & Projectile & Protons \\
\hline & Energy & $470-510 \mathrm{MeV}$ \\
\hline & Intensity & $\# 100 \mu \mathrm{A}$ \\
\hline & Beam Size & $\# 1 \mathrm{~cm}^{2}$ \\
\hline & Time Structure & $\mathrm{cW}$ \\
\hline \multirow[t]{2}{*}{ Target } & Length & \#_20 cm \\
\hline & Power Deposited & \# $20 \mathrm{~kW}$ \\
\hline \multicolumn{3}{|c|}{ RADIOACTIVE ION BEAM SYSTEM } \\
\hline \multirow[t]{2}{*}{ Ion Source } & \multicolumn{2}{|c|}{ Surface, ECR, Laser, FEBIAD, etc. } \\
\hline & Max. Energy & \# $60 \mathrm{keV}$ \\
\hline \multirow[t]{3}{*}{ Mass Separator } & Mass/ $\delta($ Mass) & \# 10000 \\
\hline & Mass & \# 240 \\
\hline & Vacuum & $\# 2 \times 10^{-7}$ torr \\
\hline \multicolumn{3}{|c|}{ ACCELERATOR SYSTEM } \\
\hline \multirow[t]{2}{*}{ Pre-Buncher } & $\begin{array}{l}\text { Fundamental } \\
\text { Frequency }\end{array}$ & $11.67 \mathrm{MHz}$ \\
\hline & Harmonics & 3 \\
\hline \multirow[t]{4}{*}{ RFQ } & Input Energy & $2 \mathrm{keV} / \mathrm{amu}$ \\
\hline & $\begin{array}{l}\text { Input } \\
\text { charge/mass }\end{array}$ & $\exists 1 / 30$ \\
\hline & Output Energy & $150 \mathrm{keV} / \mathrm{amu}$ \\
\hline & Frequency & $35 \mathrm{MHz}$ \\
\hline \multirow[t]{4}{*}{ DTL } & Charge/mass & $\exists 1 / 6$ \\
\hline & Output Energy & $\begin{array}{l}0.15 \text { to } 1.5 \\
\mathrm{MeV} / \mathrm{amu}\end{array}$ \\
\hline & Frequency & $105 \mathrm{MHz}$ \\
\hline & $\Delta \mathrm{E} / \mathrm{E}$ & $<0.1 \%$ \\
\hline
\end{tabular}

\section{ISAC CIVIL CONSTRUCTION}

The first major ISAC-I construction contract (for the relocation of site services) was awarded in May 1996 followed by the primary structure contract commencing in September 1996. In July 1997, TRIUMF personnel began occupying the experimental hall for installation of experimental apparatus. Figure 1 is a sketch of the building layout showing through cut out sections the main features 
of ISAC-I. The main elements of the facility include the following; (a) a bored hole through the cyclotron vault wall and an underground tunnel to transport the high intensity proton beam from the cyclotron to the ISAC target stations, (b) a four story target service wing containing a chemistry laboratory, an assembly laboratory, a meeting room, service elevator, active sumps and two open areas for offices, (c) a heavily shielded target vault containing two target stations, shielding for two $50 \mathrm{~kW}$ beam dumps, two hot cells, a target storage pit, all of which can be accessed with a remotely controlled 20 ton rolling crane, (d) a penthouse above the target vault housing the HEPA filtered exhaust system, the building mechanical services and the heat exchanger, (e) a shielded room for a high resolution mass separator, (f) a grade level experimental hall for the accelerators and experimental stations with a 35 ton rolling crane, (g) a $250 \mathrm{~kW}$ diesel generator for emergency power, (h) two experimental counting rooms and (i) an accelerator control room.

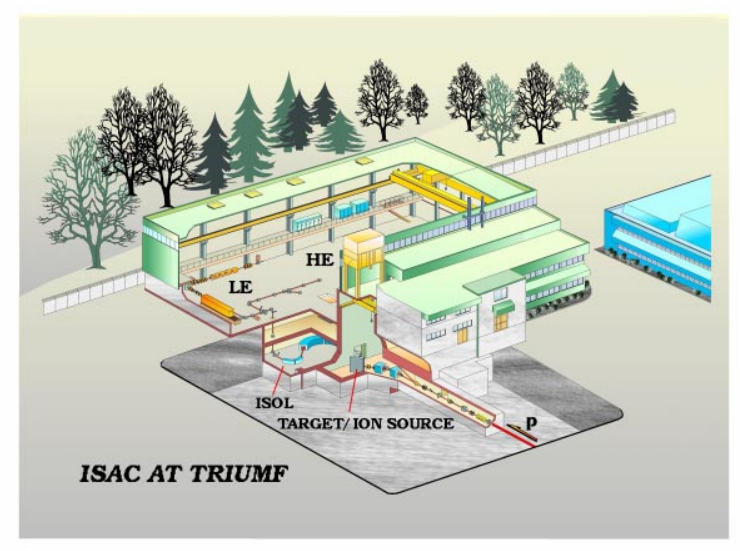

Figure 1. A sketch of the ISAC-I facility showing the proton beam line, the target vault, the target service building, the mass separator vault, the TRINAT mezzanine (above the mass separator vault) and the accelerator/experimental hall.

\section{ISAC TECHNICAL FACILITY}

\subsection{Proton Beam Line}

A new beam line was built to take the proton beam from the cyclotron to the ISAC-I target. In May 1997, proton beams were extracted from the cyclotron into the front end of the beam line over the energy range 472 to 510 $\mathrm{MeV}$. The entire beam line was completed to the ISAC-I beam dump by the spring 1998 . This culminated with the timely completion of a major milestone, namely, beam to the ISAC beam dump in May 1998.

\subsection{Target}

The target hall vault has three main areas; a target area, a storage area and a hot cell area. The entire vault is serv- iced by a 20 ton overhead crane that can be operated remotely from the target service building. In order to achieve efficient ISAC operation, it was necessary to shield the target area such that personnel could work in the target vault during beam operation. The required shielding was estimated assuming a $100 \mu \mathrm{A}$ proton beam impinging on a high $\mathrm{z}$ target such as uranium. The shielding surrounds two, large, T-shaped, steel vacuum-tanks which each contain five removable modules. Two of the modules (an entrance module that contains proton beam diagnostics and an exit module that houses the proton beam dump) are required for the proton beam. The remaining three modules (a target module which holds the target, ion source and ion beam extraction electrodes and two exit modules which contain the ion beam optical elements and the ion beam diagnostics) are required for the RIB. These three modules have all of the ion optical elements placed inside of a primary vacuum vessel within the secondary vacuum space of the T-shaped tank. The dual vacuum system is designed to prevent radioactive contamination from migrating to either the outside walls of the modules or to the entrance and dump modules. The entrance and dump modules are located in the secondary vacuum of the large tank. Water-cooled windows are used where the proton beam enters and exits the target module. The volatile compounds that are pumped from the target tank are stored in tanks, monitored for activity and allowed to decay to acceptable levels prior to release to the atmosphere through HEPA filters. Although both target tanks were manufactured, initially only the west target tank and five modules are presently operational. The second tank is used to condition, store and leak check spare modules. The servicing philosophy of the components in the tanks is based on years of successful experience with the meson production targets. The services are manually disconnected from a module requiring servicing and then the overhead crane is used to pick up that module and transport it to either a storage vessel or to the hot cell where manipulators are used to remove and replace components. Details of the target station are found elsewhere in these proceedings [2].

Initially the beam current on target was only about one microampere. During this stage it was necessary to provide ohmic heating in order to achieve the required target temperature (about $1500^{\circ} \pm 100^{\circ} \mathrm{C}$ ). These targets are similar to those presently used in TISOL. As the proton beam current (beam power) is increased it will eventually be necessary to cool the target.

\subsection{Ion Source}

The operational target and ion-source system requires frequent servicing to meet the experimental requests for isotopes. The first ion source to be approved for ISAC-I experiments is a surface ion source for alkali elements. A double-gap extraction system was designed and tested. The beam could be extracted over a wide range of masses 
and energies without intensity or emittance degradation. As predicted by the simulations, it was unnecessary to adjust the electrode position each time a new mass or new ion energy was selected. The ion source and target have been designed to operate up to a maximum bias of $60 \mathrm{kV}$. The first experiment approved by the TRIUMF Experimental Evaluation Committee (EEC) required ${ }^{37,38 \mathrm{~m}} \mathrm{~K}$ beams. ISAC used a compressed $\mathrm{CaO}$ pellet target to produce the potassium isotopes. Other isotopes requiring different ion sources are required quickly. An electroncyclotron-resonance (ECR) ion source has been very useful in ionising gaseous elements in TISOL. Development has started on a compact microwave ECR ion source. ECR ion sources produce ions with energy spreads of tens of $\mathrm{eV}$. It is not possible to achieve a mass resolution (isobaric rejection) greater than 10,000, unless the energy spread is kept below about $2 \mathrm{eV}$. The initial experiments, requiring an ECR ion source, have not requested a high mass-resolution. Provision has been made so that the ISAC system can accommodate laser ion sources, FEBIAD ion sources and Cusp ion sources. These sources will have to be developed for ISAC as required by the scientific programs.

\subsection{Mass Separator}

The ISAC mass separator system includes a pre-separator magnet, three matching sections using electrostatic optics, an acceleration column, a mass analysing magnet and a deceleration column. The ion optics were calculated using the computer code GIOS which was modified to predict the effect of the acceleration columns. The pre-separator stage uses a $>\mathrm{Y}=$ shaped magnet that is designed to accept the RIB from either one of the two target stations. Its purpose is to select out most of the unwanted radioactive ions and to deposit this activity on slits that can be removed remotely to the hot cells for servicing. The mass separator-magnet was acquired from Chalk River in August 1997, following the closure of the TASCC laboratory. The separator is located on a high voltage platform that will eventually raised in potential up to $60 \mathrm{kV}$. By varying the potential of the mass separator it will be possible to scan and select a particular isotope without readjusting the magnetic field of the magnet. The system has been designed to provide a mass resolving power of 10,000 for an emittance of $30 \pi \mathrm{mm} \mathrm{mrad}$. An outline drawing showing the configuration of the mass separator system is given in figure 2 .

\subsection{Low Energy Beam Transport}

The ion beam from the mass separator can be transported either to the low energy experimental area or to the RFQ accelerator. At the same time, it is desirable to have an off-line ion source (OLIS) to provide a beam of stable isotopes to the same two areas, although its primary purpose is for commissioning the accelerators. A switchyard has been designed and built to meet these goals. All of the optics in the low-energy-beam-transport (LEBT) is electrostatic. The line is made up of a number of similar modules. Spherical bends are used to achieve focusing in both transverse planes. The RFQ, having no bunching section, requires an external buncher in the LEBT. The section of the LEBT that connects the mass separator to the rest of the low energy beam line was assembled for the October 1998 beam commissioning to TRINAT. The LEBT from the OLIS to the RFQ was commissioned early in 1998. The installation of the OLIS began in July 1997 and the first ion beam was extracted in November of the same year. The LEBT and buncher were commissioned from OLIS to the entrance of the RFQ by March 1998.

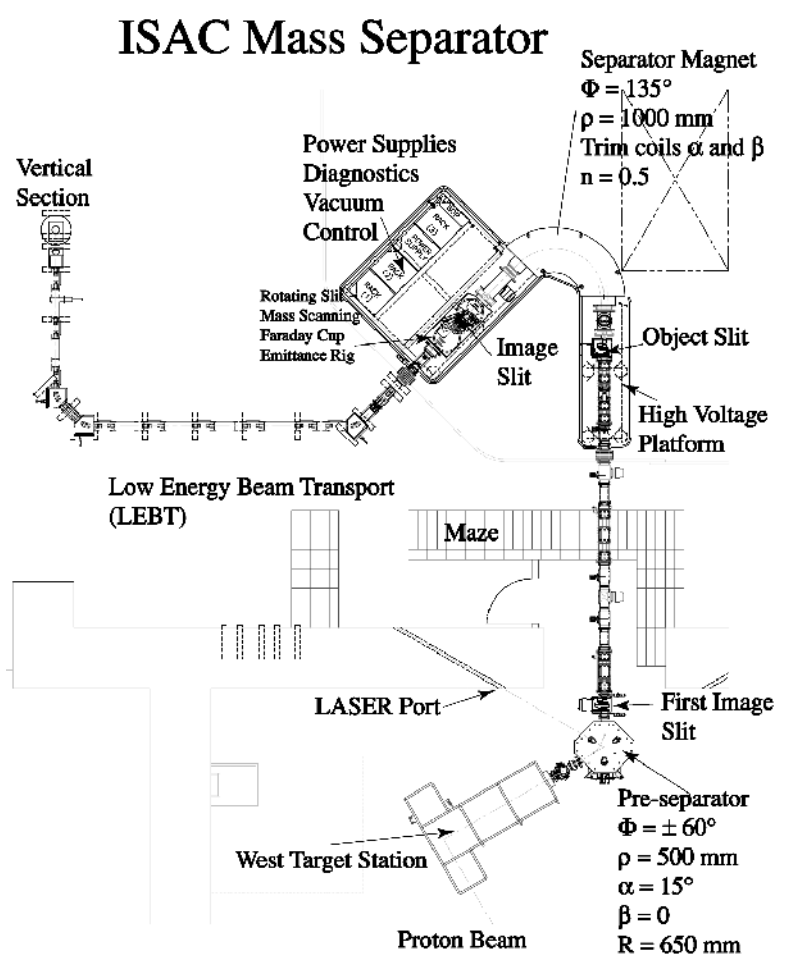

Figure 2. Layout of the beam transport system at the target elevation from the target station through the mass separator system to the LEBT

\subsection{Accelerators}

Beams having a mass to charge ratio less than or equal to 30 are to be accelerated from the injection energy of 2 $\mathrm{keV} / \mathrm{amu}$ up to a final energy of $1.5 \mathrm{MeV} / \mathrm{amu}$. The accelerating system consists of a pre-buncher, a cw RFQ, a medium energy beam transport (MEBT) section, an electron stripper, a re-buncher, and a cw drift tube linac with three split ring bunchers [3],[4],[6],[7]. The pre-buncher provides a pseudo saw tooth velocity profile at a fundamental frequency of $11.67 \mathrm{MHz}$, thereby providing approximately $86 \mathrm{nS}$ between beam bursts. Bunched beam from the pre-buncher fills every third bucket of the 35 $\mathrm{MHz}, \mathrm{cw}, 8 \mathrm{~m}$ long RFQ. The beam out of the RFQ, at energy $0.15 \mathrm{Mev} / \mathrm{amu}$, is first focused and stripped to 
higher charge states. The beam is then magnetically bent to select only those isotopes having a mass to charge ratio less than or equal to 6 and then re-bunched in a $35 \mathrm{MHz}$ spiral re-buncher prior to injection into the first tank of the DTL. The DTL must provide a bunched beam that can be continuously varied in energy from 0.15 to $1.5 \mathrm{MeV} / \mathrm{amu}$. To achieve this a separated-function structure with five DTL tanks and three split-ring bunchers has been designed. As the DTL and buncher system operates $\mathrm{cw}$ at $105 \mathrm{MHz}$, only 1 in 9 rf buckets are used to accelerate beam.
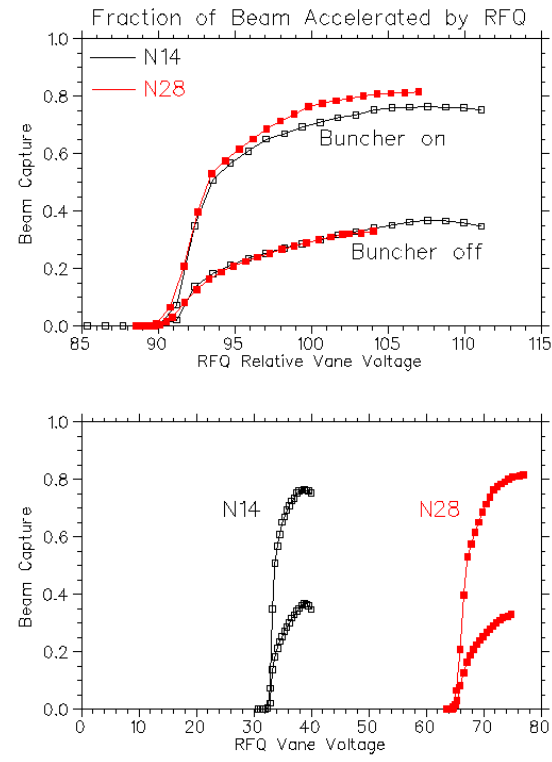

Figure 3. Initial transmission of atomic and molecular nitrogen ion beams through the 7 ring RFQ with and without pre-buncher as a function of vane voltage.

In order to demonstrate that the RFQ would operate $\mathrm{cw}$ at $35 \mathrm{MHz}$, a $1 \mathrm{~m}$ long (3 rings) prototype was successfully tested without beam but at full power in November of 1996. The RFQ accelerator requires precise alignment to operate with the predicted acceptance. In order to demonstrate that the required alignment had indeed been achieved and that the numerical simulations were correct, a RFQ tank with only the first 7 of the eventual 19 rings was commissioned with beam in 1998 [8]. Beams of stable nitrogen isotopes were produced in the off line ion source and accelerated through the RFQ to a diagnostic station. The ion source provided both atomic (mass 14) and molecular (mass 28) ions. The beam transmission and acceptance of the RFQ were found to be in excellent agreement with predictions. In figure 3 the transmission through the RFQ is shown as a function of the two masses as a function of vane voltage.

A prototype $35 \mathrm{MHz}$ spiral re-buncher was built in 1998 and has been tested at signal level [5]. The operational spiral buncher should be ready for installation by the end of 1999. The first tank of the DTL with electrodes has been manufactured and tested at full power. The remaining four tanks will be sent out for manufacture in 1999 with the second tank ready for installation early in 2000. The first DTL split-ring buncher was built in collaboration with the Institute for Nuclear Research by the rf group at INR, Troitsk, Russia [6]. This buncher was delivered to TRIUMF in August 1998 and subsequently tested successfully at full power. This group is now constructing the remaining two bunchers. Detail design has started on the four magnetic triplets required to achieve transverse focusing between each DTL tank. Figure 4 locates the accelerator systems within the $30 \mathrm{~m}$ wide by $64 \mathrm{~m}$ long experimental hall.

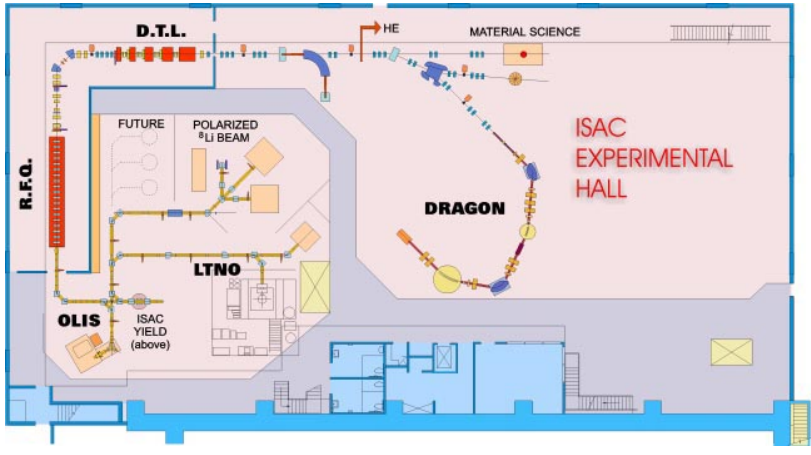

Figure 4. The layout of the experimental/accelerator hall in ISAC-I shows the locations of the accelerators and the various experimental stations. The lifetime station is located to the right of the yield station.

\subsection{ISAC Science Facilities}

The initial experimental program at ISAC includes several experimental stations. Space in the experimental hall in ISAC has been allocated to accommodate these facilities. In particular, the experimental stations include; the TRIUMF Neutral Atom Trap (TRINAT), a Yield Station, the Low Temperature Nuclear Orientation system (LTNO), a General Purpose \& Lifetime measurement station, the recoil mass spectrometer (DRAGON) facility and a ${ }^{8} \mathrm{Li}$ polarizer for the $\beta$-NMR station. The hall can accommodate additional stations. In particular, it is anticipated that the Canadian Penning Trap will move to ISAC when beams of interest become available. TRINAT is located below grade in a well-shielded mezzanine above the mass separator. The layout of the stations is given in figure 4.

TRINAT has been trapping radioactive atoms at the TISOL facility in a Zeeman optical trap. Detection of the decay products from these trapped atoms permits sensitive tests of the Standard Model. The trap was recently moved from TISOL to ISAC where the higher beam flux should yield increased sensitivity. In nuclear astrophysics, many of the reactions of interest involve proton or alpha captures. The ISAC nuclear astrophysical experiments will be carried out with reverse kinematics by bombarding a 
high-pressure windowless gas-target with the accelerated radioactive ion beams. The reaction products are detected using a recoil mass fragment detector, DRAGON, designed to have a high detection-selectivity at the ISAC energies. The nuclear orientation facility (LTNO) has been shipped from ORNL to TRIUMF and has been reassembled in the low energy experimental area of ISAC. The initial scientific program for the LTNO will focus on nuclear structure in the mass range 80 to 100 . In addition the facility will use nuclear magnetic resonance on oriented nuclei (NMRON) as a sensitive probe of condensed matter. The $\mu$ SR group at TRIUMF is building a $\beta$-NMR facility that initially will be using a polarised ${ }^{8} \mathrm{Li}$ beam to enhance their condensed matter program at TRIUMF. The longer lifetime of the radioactive isotope, compared to that of the muon, makes it the preferred probe for relaxation studies in solids.

\section{COMMISSIONING/OPERATION}

A $1 \mu \mathrm{A}$ proton beam has been transported from the cyclotron to the ISAC beam dump. It is expected that a target will be ready to permit a full power test at $100 \mu \mathrm{A}$ by December 1999. A beam of stable isotopes was delivered to TRINAT in October 1998. In November the proton beam was used to produce a radioactive potassium beam from a $\mathrm{CaO}$ target for TRINAT and the ISAC lifetime station. Operation of the low energy beam will expand to the other low-energy experimental-stations as they become available in 1999. The off-line ion source allows the RFQ, MEBT, DTL and HEBT commissioning to take place at the same time as the radioactive beam is being delivered to the low energy experiments. The schedule anticipates that by the end of 2000 the accelerators will be operating and that the high-energy experiments will be ready for an accelerated radioactive ion beam.

\section{FUTURE PLANS}

The five-year funding cycle, which was used to build ISAC-I, began in June 1995 and ends in April 2000. A new proposal for the next five years was prepared for submission to the Canadian Government in November 1998. In response to TRIUMF=s request for input into the new five-year plan, the Canadian user community made a strong submission to have ISAC-I augmented up to an energy of $6.5 \mathrm{MeV} / \mathrm{amu}$ for masses less than or equal to 150. These submissions were used to produce some generalised facility specifications [9]. . Additionally, a request to provide slightly higher energies for the lighter masses is possible with this design. The proposed ISACII facility is shown in figure 5. The design would use a charge state booster in the LEBT before the RFQ and accelerate the isotopes in a new DTL to $400 \mathrm{keV} / \mathrm{amu}$ before further stripping the heavier masses to higher charge states for acceleration through a superconducting linac. A modest grade-level building expansion will be required to ac- commodate the additional accelerators and experimental stations. Construction of ISAC-II could be staged in order that the nuclear physics program can begin prior to facility completion. To achieve this early start to an experimental program, it is proposed to carry out the building construction concurrently with the assembly of small superconducting rf cavities. These cavities would then be initially installed, as they become available, downstream of the DTL1 in the ISAC-I experimental hall.

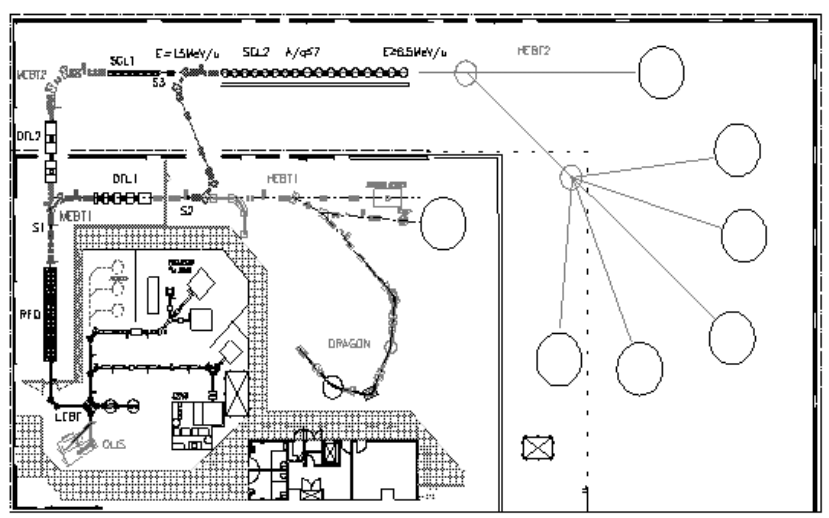

Figure 5. This proposed layout for ISAC-II shows how the accelerators and experimental stations might be added to ISAC-I.

\section{REFERENCES}

[1]J.M. D'Auria et al., Nuclear Instruments and Methods in Physics Research B 126, 7-11 (1997)

[2]P. Bricault et al., "A $500 \mathrm{MeV} 100$ micro Amp proton Target for ISAC Radioactive Ion Beam Facility", these proceedings, MODR4

[3]R.L. Poirier et al., "RF Systems of the ISAC Facility", these proceedings, THBL4

[4]M. Laverty et al., "Design and Testing of the TRIUMF ISAC RFQ Control System", these proceedings, MOP94

[5]A.K. Mitra, R.L. Poirier, "A 35 MHz Spiral ReBuncher Cavity for the TRIUMF ISAC Facility", these proceedings, MOP75

[6]Y.V. Bylinsky et al., "High Power Test of the ISAC Triple Gap Buncher Operating in CW mode", these proceedings, MOP95

[7]P. Bricault et al., "Initial commissioning of the DTL Tank1 of the ISAC LINAC", these proceedings, FRA66

[8]R.E. Laxdal et al., "Final Beam Test Results with the ISAC $35 \mathrm{MHz}$ RFQ", these proceedings, A08

[9]R.E Laxdal, R.A. Baartman, "Design Optimization of the proposed ISAC-2 Project at TRIUMF", these proceedings, FRA65 